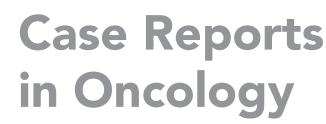

Case Reports

in Oncology

\title{
Ambulatory Intrapleural Fibrinolytic Therapy in Highly Viscous Recurrent Malignant Pleural Effusion
}

\author{
Chuan Tai Foo Jurgen Herre \\ Department of Respiratory Medicine, Cambridge University Hospitals NHS Foundation \\ Trust, Cambridge, UK
}

\author{
Keywords \\ Intrapleural fibrinolytic therapy · Lung cancer · Malignant pleural effusion · Viscous pleural \\ effusion
}

\begin{abstract}
Malignant pleural effusion (MPE) is common in lung cancer. We report a case of highly viscous recurrent MPE associated with lung cancer. The viscosity prohibited gravity-dependent drainage initially with a $6-\mathrm{Fr}$ aspiration catheter and subsequently with a $12-\mathrm{Fr}$ intercostal drain. The effusion was eventually evacuated after a single dose of intrapleural fibrinolytic therapy. This process was repeated a total of 13 times over a 12-month period in an ambulatory setting. No bleeding complications were observed. This case highlights the feasibility and safety of repeated intrapleural fibrinolysis in the management of highly viscous recurrent MPE in an ambulatory setting.

\section{Introduction}

Malignant pleural effusion (MPE) is common in lung cancer. This often appears haemoserous or haemorrhagic and can be drained using a range of devices including aspiration catheters, intercostal chest drains, and indwelling pleural catheters (IPCs). Highly viscous MPE is uncommon, with limited treatment options available. We present a case of a highly viscous recurrent MPE successfully managed with repeated ambulatory intercostal chest drain and intrapleural fibrinolytic therapy (IPFT). 

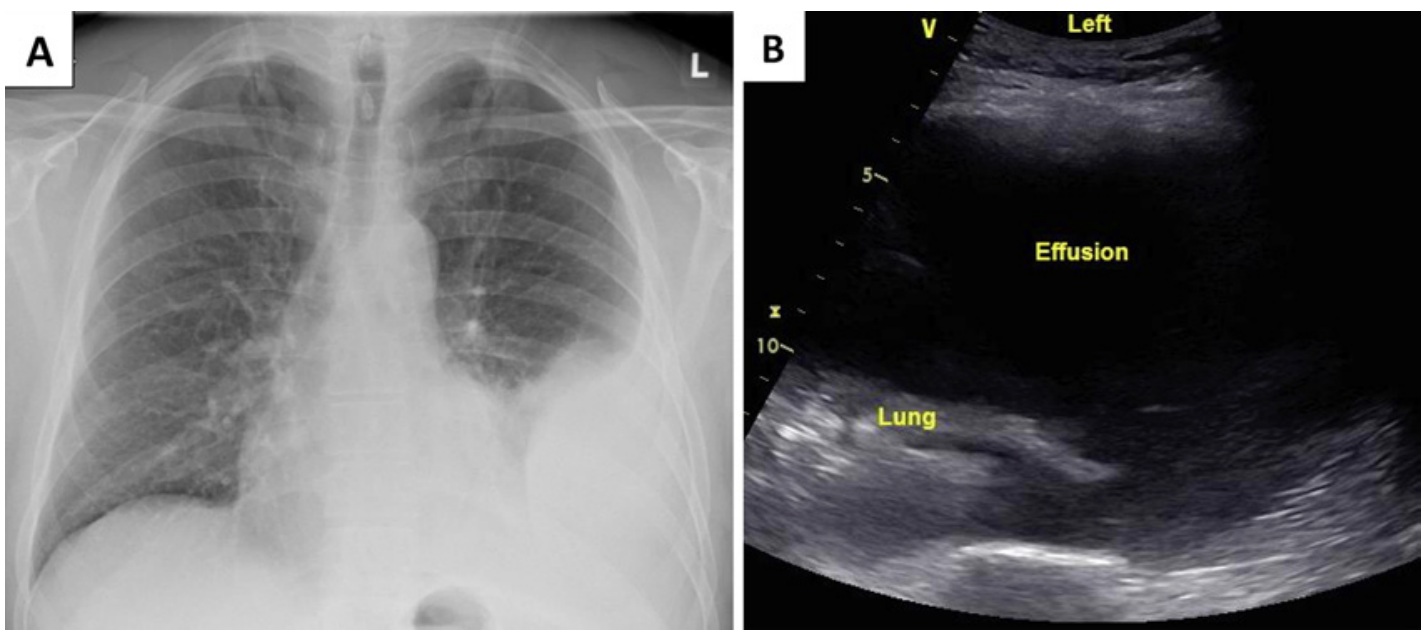

Fig. 1. Chest X-ray (A) and thoracic ultrasound (B) images showing a large left-sided pleural effusion.

\section{Case Report}

A 54-year-old male with no significant past history presented with exertional dyspnoea, cough, and small-volume haemoptysis. On history, the patient was a lifelong non-smoker and reported no infective or constitutional symptoms. No occupational or environmental exposures were identified. Chest X-ray showed a large left-sided effusion that was aspirated. Cytology and immunohistochemical stains confirmed lung adenocarcinoma. Staging computed tomography (CT) revealed T2aN2M1b disease with metastatic deposits along the pleural and in the liver. Activating epidermal growth factor receptor (EGFR) mutation (exon 19 deletion) was detected and afatinib was commenced. Thoracoscopic talc poudrage pleurodesis and transient IPC insertion was also undertaken. The effusion resolved subsequently and the IPC was removed. Serial chest CT at 6 months demonstrated partial response to immunotherapy, but repeat CT at 12 months showed disease progression. EGFR T790M mutation was detected and afatinib was switched to osimertinib. Around the same period, the patient became symptomatic with recurrence of the left-sided pleural effusion. This was therapeutically aspirated to dryness using a 6-Fr thoracentesis catheter and revealed thick exudative pleural fluid. A month later, the effusion recurred and, due to its increased viscosity, necessitated evacuation using a 12-Fr intercostal drain (ICD). Subsequent drainage in the clinic 4 weeks later was again attempted through a temporarily placed 12-Fr ICD but was incomplete as the fluid had become highly viscous (Fig. 1). In an attempt to reduce fluid viscosity and promote further drainage, $5 \mathrm{mg}$ dornase alfa and $10 \mathrm{mg}$ alteplase were administered intrapleurally via the ICD. Following a 2-h dwell time, a total of 2,000 mL of fluid was drained. Postdrainage chest X-ray and thoracic ultrasound showed only minimal residual effusion and the patient was discharged home the same day (Fig. 2). This process was repeated in an ambulatory fashion for a total of 13 times over the next 12 months during which serial CT showed stable disease. An IPC was eventually inserted after subsequent drainages had demonstrated a significant reduction in pleural fluid viscosity and intrapleural enzymes were no longer required to assist in fluid removal. Six months later, the patient's disease progressed and a new chemo-immunotherapy regimen was commenced. Shortly after he became neutropaenic and developed an empyema necessitating IPC removal. Since then, the patient has remained stable with adequate control of his malignancy and no further re-accumulation of pleural fluid. No complications from intrapleural enzymes were noted following any of the administrations. 

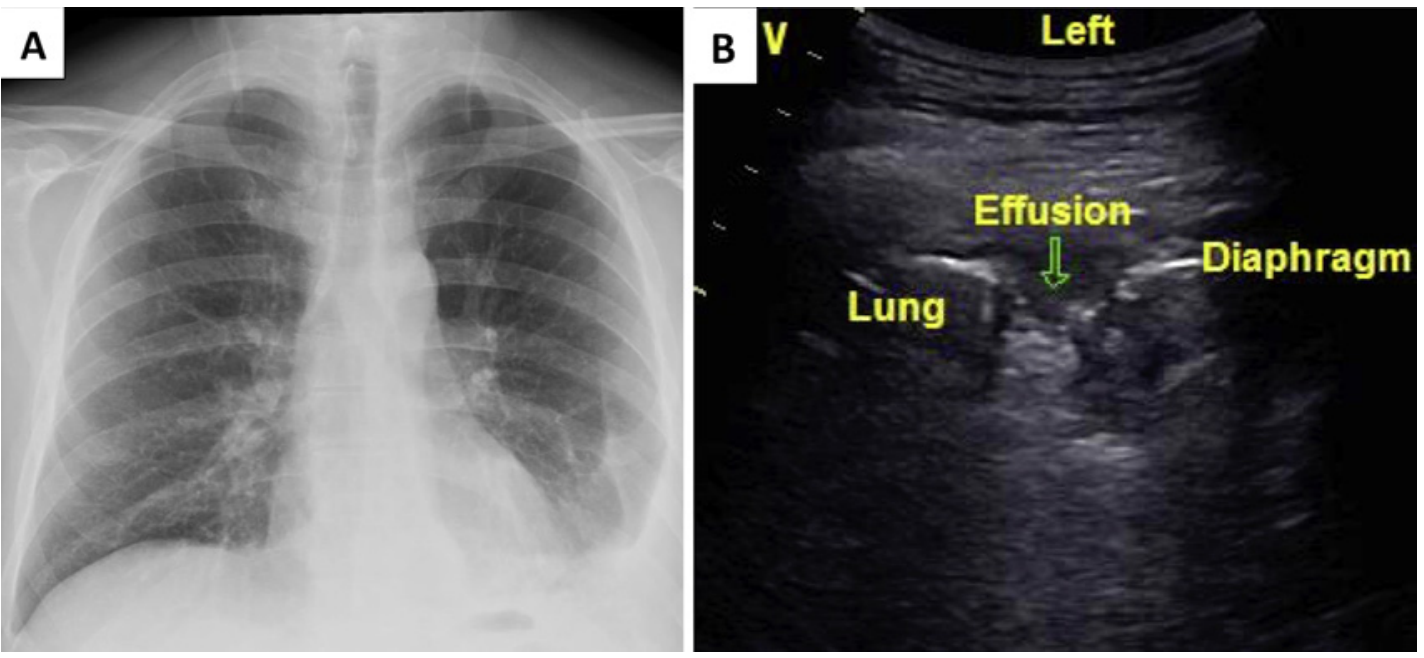

Fig. 2. Chest X-ray (A) and thoracic ultrasound (B) images showing near-complete resolution of the leftsided pleural effusion post intrapleural fibrinolytic therapy and drainage.

\section{Discussion and Conclusion}

The goal in the management of MPE is to relieve dyspnoea and allow the patient to live a life as close to "normal" as possible. Ideally this should be achieved in the least invasive manner while minimising the number of repeated procedures and interactions with the healthcare system [1]. Current available options include repeated thoracentesis, pleurodesis, and insertion of an IPC, each of these options having its own disadvantages [2].

Pleural fluid viscosity is influenced by cellular constituents and macromolecule composition, with protein being the major component [3]. Highly viscous pleural effusions are generally due to an exudative process such as infection or malignancy [4]. The increased viscosity often presents a management challenge due to the difficulty in evacuating it from the pleural cavity, as shown in this case. Presently there are limited clinically available options for viscous MPE. Hyaluronidase has been shown to be effective at reducing fluid viscosity of effusions rich in hyaluronic acid, but only in ex vivo experiments [5]. Although the use of IPFT has been widely described in patients with parapneumonic effusions, empyema, and loculated MPE, its utility in the management of viscous MPE remains unclear [2, 6].

In our report, we describe the repeated successful management of a highly viscous recurrent MPE utilising ambulatory ICD and IPFT over a 12-month period. Our patient had an incomplete pleurodesis due to a partly trapped lung and an IPC was not undertaken initially for the following reasons: Firstly, our patient had a highly active lifestyle and was frequently training for endurance events and found the prospect of an IPC unappealing. Secondly, the rate of fluid re-accumulation was slow and drainage was only required every 3-4 weeks. The exact role of IPFT in promoting fluid removal in our case remains unclear. Dornase alfa has been shown to reduce pleural fluid viscosity and promote drainage in infected effusions by the degradation of extracellular deoxyribonucleic acid, but has not been well studied in MPE [7]. Alteplase, on the other hand, is known to stimulate production of pleural fluid in both healthy and diseased pleura [8]. While it is plausible that the dilutional effect of this fluid formation helped with promoting drainage, its impact on the viscosity of MPE remains unknown. Nonetheless, we have shown that IPFT can be successful in promoting fluid removal in viscous MPE and should be considered in the absence of other 
alternatives. Furthermore, the repeated use of IPFT in our case was not associated with any bleeding complications.

In summary, we report on the feasibility and safety of using repeated IPFT in the management of highly viscous recurrent MPE in an ambulatory setting. We believe that such an approach can be considered in the appropriate circumstances.

\section{Statement of Ethics}

Written informed consent was obtained from the patient for publication of this case report and any accompanying images.

\section{Conflict of Interest Statement}

The authors have no conflict of interest to declare.

\section{Funding Sources}

None.

\section{Author Contributions}

C.T. Foo was involved in the conceptualisation, data curation, writing of the original draft, and review and editing of the final manuscript. J. Herre was involved in the conceptualisation and review and editing of the final manuscript.

\section{References}

1 Bibby AC, Dorn P, Psallidas I, Porcel JM, Janssen J, Froudarakis M, et al. ERS/EACTS statement on the management of malignant pleural effusions. Eur J Cardiothorac Surg. 2019;55(1):116-32.

2 Feller-Kopman DJ, Reddy CB, DeCamp MM, Diekemper RL, Gould MK, Henry T, et al. Management of malignant pleural effusions. An official ATS/STS/STR clinical practice guideline. Am J Respir Crit Care Med. 2018;198(7): 839-49.

3 Chang LC, Hua CC, Liu YC, Chu CM, Chen HJ, Lee N. Pleural fluid viscosity may help identifying malignant pleural effusions. Respirology. 2008;13(3):341-5.

4 Yetkin 0, Tek I, Kaya A, Ciledag A, Numanoglu N. A simple laboratory measurement for discrimination of transudative and exudative pleural effusion: pleural viscosity. Respir Med. 2006;100(7):1286-90.

5 Cheah HM, Fitzgerald D, Louw A, Creaney J, Lee YCG. Hyaluronic acid in viscous malignant mesothelioma pleural effusion. Respirol Case Rep. 2021;9(1):e00694.

6 Piccolo F, Pitman N, Bhatnagar R, Popowicz N, Smith NA, Brockway B, et al. Intrapleural tissue plasminogen activator and deoxyribonuclease for pleural infection. An effective and safe alternative to surgery. Ann Am Thorac Soc. 2014;11(9):1419-25.

7 Simpson G, Roomes D, Heron M. Effects of streptokinase and deoxyribonuclease on viscosity of human surgical and empyema pus. Chest. 2000;117(6):1728-33.

8 Piccolo F, Popowicz N, Wong D, Lee YC. Intrapleural tissue plasminogen activator and deoxyribonuclease therapy for pleural infection. J Thorac Dis. 2015;7(6):999-1008. 